\title{
RECURSIVE ALGORITHMS FOR NONNORMAL PADÉ TABLES*
}

\author{
A. BULTHEEL $\dagger$
}

\begin{abstract}
The Berlekamp-Massey algorithm [L. S. De Jong, Numerical aspects of realization algorithms in linear systems theory, Ph.D. thesis, T. H. Eindhoven, the Netherlands, 1975] and [J. L. Massey, Shift-register synthesis and BCH decoding, IEEE Trans. Information Theory, IT-15 (1969), pp. 122-127] for minimal realization problems is a special case of the Padé approximation problem. As a matter of fact, it computes among other polynomials the denominators of the elements of the Padé table that are on the descending diagonal $\{[0 / 1],[1 / 2], \cdots,[k / k+1], \cdots\}$ as far as they exist and this algorithm works for nonnormal Padé tables too. This algorithm does not seem to be well-known in Padé approximation literature. It is not very difficult to generalize this algorithm so as to compute the other Padé approximants of a nonnormal table. Some variants will lead to a generalization of the algorithm of Brezinski [Computation of Padé approximants continued fractions, J. Comput. Appl. Math., 2 (1976), pp. 113-123], which computes the descending diagonals of a normal Padé table, and of the algorithm of Watson [D. Bussonnais, "Tous" les algorithmes de calcul par recurrence des approximants de Padé d'une serie, Construction de fractions continués correspondantes, Séminaire d'Analyse Numérique, No. 293, Grenoble, 1978], [G. Claessens, A new look at the Padé table and the different methods for computing its elements, J. Comput. Appl. Math., 1 (1975), pp. 141-152], [P. J. S. Watson, Algorithms for differentiation and integration, Padé Approximants and their Applications, P. R. Groves-Morris, ed., Academic Press London, 1973, pp. 93-98] that computes the descending staircases of normal tables. This work is the dual of Cordellier's [Deux algorithmes de calcul récursif des éléments d'une table de Padé non normale, Conference on Padé approximation, Lille, France, 1978], and of McEliece and Shearer [A property of Euclid's algorithm and an application to Padé approximation, SIAM J. Appl. Math., 34 (1978), pp. 611-616] deriving similar results for ascending diagonals and staircases. A more continued fraction-like approach would generalize the Thatcher algorithm and may be found in [J. A. Murphy and H. R. O'Donohoe, A class of algorithms for obtaining rational approximants to functions which are defined by power series, Z. Angew. Math. Phys., 28 (1977), pp. 1121-1131] and [A. Bultheel, Division algorithms for continued fractions and the Padé table, Applied Mathematics and Programming Division, Rep. TW41, Katholicke Universitiet Leuven, Heverlee, Belgium, August 1978].
\end{abstract}

1. Introduction. We recall some basic definitions and some well-known properties without proof.

Corresponding to a formal power series $\sum_{k=0}^{\infty} c_{k} z^{k}$ we define for all $L, M \geqq 0$ a Padé form

$$
(L / M)_{N}=\frac{P^{(L / M)}(z)}{Q^{(L / M)}(z)}
$$

as any couple of polynomials $P^{(L / M)}, Q^{(L / M)}$ (if it exists) satisfying

$$
\begin{aligned}
& \text { 1) } \left.\begin{array}{l}
d^{\circ} P^{(L / M)}=L, \\
\text { 2) } d^{\circ} Q^{(L / M)}=M,
\end{array}\right\} \text { (but the degree need not be strict), } \\
& \left.(\mathrm{PF})_{N} 3\right) P^{(L / M)} \text { and } Q^{(L / M)} \text { are relative prime, } \\
& \text { 4) } Z^{(L / M)}=Q^{(L / M)} f-P^{(L / M)}=r_{N} z^{N}+r_{N+1} z^{N+1}+\cdots \text {, } \\
& \text { 5) some normalizing condition. }
\end{aligned}
$$

If this couple does not exist then we set by convention $(L / M)_{N}=$ nill. If $(L / M)_{N} \neq$ nill and $r_{N} \neq 0$ then $N$ is called its order. $Z^{(L / M)}$ is called the residual.

Normalization can be chosen in several ways, e.g., making $P$ or $Q$ monic or comonic. Here we will choose as normalizing condition $Q^{(L / M)}(0)=1$.

* Received by the editors December 5, 1978.

$\dagger$ Afd. Toegepaste Wiskunde en Programmatie, Katholieke Universiteit Leuven, Celestijnenlaan 200 A, B-3030 Heverlee, Belgium. 
Strictly spoken, there is a difference between, e.g.,

$$
\frac{1}{1} \text { and } \frac{1+0 z}{1+0 z+0 z^{2}}
$$

because the first is an element of the form $(0 / 0)_{N}$ and the second is of the form $(1 / 2)_{N}$. We will go rather loosely over this subtle difference and regard them as equal.

If the order of a Padé form $(L / M)_{N}$ is at least $L+M+1$ then it is called a Padé approximant and it is denoted by $[L / M]$. As is well known: a Padé approximant is uniquely defined. $\{L / M\}$ is the set of all Padé forms of some arbitrary order

$$
\{L / M\}=\left\{(L / M)_{N} \mid N=0,1,2, \cdots\right\} .
$$

Its elements are nill and all the rational forms that are reduced, normalized and with $d^{\circ}$ numerator $\leqq L$ and $d^{\circ}$ denominator $\leqq M$.

PROPERTY 1.

$$
\{I / J\} \subset\{L / M\} \quad \Leftrightarrow \quad I \leqq L \text { and } J \leqq M .
$$

A Padé table is a tableau containing in its $L$ th row and $M$ th column the element $[L / M]$.

A Padé table is normal if all $[L / M]$ are different from nill (and are different from each other).

The structure of a nonnormal Padé table is well-known (see e.g., the example in Gragg [8, p. 12]). It is divided into square blocks of size $n \times n(n \geqq 1)$. If $n>1$ then the block is a singular block and a singular block contains up and above its antidiagonal nonnill Padé approximants that are all equal to the left top element. Below the antidiagonal all the entries are nill.

The left top element of a (singular) block will be called the indicator of the (singular) block and of its elements. The Padé table of a series

$$
c_{n} z^{n}+c_{n+1} z^{n+1}+\cdots \quad\left(n>0, c_{n} \neq 0\right)
$$

contains such a $n \times n$ block in its left top corner. To the right of it we find a band of size $n \times \infty$ with nill all over. To avoid difficulties with this band, we suppose that the leading coefficient $c_{0} \neq 0$ in the following. This is no restriction really since for $L \geqq n$,

$$
[L / M]_{z^{n} f(z)}=z^{n}[L-n / M]_{f(z)} .
$$

Property 2.

$$
c_{0} \neq 0 \Rightarrow[k / 0] \text { and }[0 / k] \text { are nonnill for all } k \text {. }
$$

The order $N$ of $\{L / M\}$ is the highest possible order that can be obtained by an element of $\{L / M\}$.

A Padé form $(L / M)_{N} \in\{L / M\}$ having this maximal order is called an order representant of $\{L / M\}$. This definition makes sense only if not all elements of $\{L / M\}$ are nill. By Property 1 we have that $\{L / M\}$ will contain among others the entries of the Padé table that are on or within the rectangle that has as diagonal $[0 / 0]-[L / M]$, especially $[L / M],[L / 0]$ and $[0 / M]$. The last two being nonnill by Property 2 .

Property 3. The order of $\{L / M\}$ is at least $\max (L, M)+1$.

From Theorem 3,2 in [8, p. 13] it may be found that the following is true.

PROPERTY 4. The order of $\{L / M\}$ is the order of the indicator of $[L / M]$.

If $[L / M]=$ nill then we call $\{L / M\}$ degenerate (i.e., $(L / M)$ indicates the position in a singular block below the antidiagonal.) 
PROPERTY 5. $\{L / M\}$ is nondegenerate iff its order is at least $L+M+1$.

Since a Padé approximant is uniquely defined, it follows from Property 4 that the following is true.

PROPERTY 6. The order representant of $\{L / M\}$ is unique and is some Padé approximant.

It is known that in the normal case the elements of a descending staircase $T_{k}$ can be computed recursively by the following relations:

Let $R^{(1)}, R^{(2)}$ and $R^{(3)}$ be three successive elements from a staircase and let

$$
\begin{aligned}
& S^{(i)}=\alpha P^{(i)}+\beta Q^{(i)}, \\
& \alpha, \beta \text { arbitrary expressions, }
\end{aligned}
$$

(e.g., $(\alpha, \beta)=(1,0)$ iff $S^{(i)}=p^{(i)} ;(\alpha, \beta)=(0,1)$ iff $S^{(i)}=Q^{(i)}$ and $(\alpha, \beta)=(-1, f(z))$ iff $\left.S^{(i)}=Z^{(i)}\right)$. Then we have [4], [5]

$$
S^{(3)}=S^{(2)}-r_{2} r_{1}^{-1} z S^{(1)},
$$

where $r_{i}$ are the leading nonzero coefficients of $Z^{(i)}$. These coefficients can be computed by

(2) a) previous recursions of the form (1) for $S^{(i)}=Z^{(i)}$

b) expressions of the form

$$
r_{2}=\sum_{\omega} b_{\omega}^{(2)} c_{N-\omega}
$$

where $Q^{(2)}=\sum_{\omega} b_{\omega}^{(2)} z^{\omega}$ and $Z^{(2)}=O\left(z^{N}\right)$.

The algorithm of Watson [4], [5], [12] uses relations (1) and (2b) and the Thatcher algorithm [4], [5] uses (1) and (2a) in an unnormalized form. There also exist similar recursions between adjacent elements of a descending diagonal

$$
S^{(3)}=\left(1+F_{1} z\right) S^{(2)}+F_{2} z^{2} S^{(1)}
$$

with

$$
F_{1}=r_{2}^{\prime} r_{2}^{-1}-r_{1}^{\prime} r_{1}^{-1}, \quad F_{2}=-r_{1} r_{2}^{-1},
$$

where $Z^{(i)}=r_{i} z^{N_{i}}+r_{i}^{\prime} z^{N_{i}+1}+\cdots$ is of order $N_{i}$.

These $r_{i}$ and $r_{i}^{\prime}$ can again be expressed in terms of $b_{\omega}^{(i)}$ and $c_{\omega}$.

This is known as Brezinski's algorithm [1].

To generalize these algorithms to the nonnormal case, we can see that the elements of such a nonnormal staircase (diagonal) may be nill or repeat themselves. Moreover the order of the approximant and the degrees of numerator and denominator are not precisely known a priori. So we will need a more complicated notion of a staircase, order and degree. This is done in the next section.

2. Diagonals, staircases, order and degree. A diagonal $\mathbf{D}_{k}$ is an ordered set consisting of the elements

$$
\{\{k / 0\},\{k+1 / 1\}, \cdots,\{k+l / l\}, \cdots\} \text { for } k \geqq 0
$$

and

$$
\{\{0 /-k\},\{1 /-k+1\}, \cdots,\{l /-k+l\}, \cdots\} \text { for } k<0 .
$$

Selecting only one Padé form (the Padé approximant) for every $\{L / M\} \in \mathbf{D}_{k}$, we will get an ordered set of Padé forms (Padé approximants) which will be referred to as 
$\hat{D}_{k}\left(\hat{D}_{k}\right)$. If we get rid of all the nill elements and the duplicate elements in $\hat{D}_{k}\left(\hat{D}_{k}\right)$ we will denote this as $D_{k}\left(D_{k}\right)$. This means that $D_{k}$ contains exactly one element for every (singular) block it passes through, viz., its indicator. By a (descending) staircase $\mathbf{T}_{k}$ we mean the ordered set

$$
\{\{k / 0\},\{k+1 / 0\},\{k+1 / 1\},\{k+2 / 1\}, \cdots,\{k+l / l\},\{k+l+1 / l\}, \cdots\} \quad \text { if } k \geqq 0
$$

and

$$
\{\{0 /-k\},\{0 /-k+1\},\{1 /-k+1\}, \cdots,\{l /-k+l\},\{l /-k+l+1\}, \cdots\} \text { if } k<0 .
$$

There is clearly a discontinuity in this definition for $k=0$ because the staircase

$$
\{\{0 / 0\},\{0 / 1\},\{1 / 1\},\{1 / 2\}, \cdots\}
$$

is not covered. So let us call this one $\mathbf{T}_{-0}$ and the previously defined $\mathbf{T}_{0}$ will be called $\mathbf{T}_{+0}$ from now on. Thus we will always suppose that the indices of $\mathbf{T}_{k}$ will be taken from the ordered set $\{\cdots,-2,-1,-0,+0,1,2, \cdots\}$ and $\mathbf{T}_{-0} \neq \mathbf{T}_{+0}$ but +0 and -0 will coincide in every other appearance. We will often give a proof of some property for $k \geqq+0$ only because mutatis mutandis the proof for $k \leqq-0$ will be the same.

Naturally $\hat{T}_{k}, \underline{T}_{k}, \hat{T}_{k}$ and $T_{k}$ are defined similarly to $\underline{\underline{D}}_{k}, \underline{D}_{k}, \hat{D}_{k}$ and $D_{k}$. As is seen from the definition $\mathbf{T}_{k}$ is an interleaving of $\mathbf{D}_{k}$ and $\mathbf{D}_{k+1}$ for $k \geqq+0$ and of $\mathbf{D}_{k}$ and $\mathbf{D}_{k-1}$ for $k \leqq-0$.

Because we do not want to make this double reference always, we will refer to $\mathbf{D}_{k}$ as the inner diagonal of $\mathbf{T}_{k}$ and to the other as the outer one.

An element $\{L / M\} \in \mathbf{T}_{k}$ will always be an element of a neighboring staircase too, which is closer to $\mathbf{D}_{0}$ or not, depending on whether $\{L / M\}$ is on the inner or on the outer diagonal of $\mathbf{T}_{k}$.

If $\{L / M\} \in \mathbf{T}_{k}$ then we will say that its degree is

$$
\begin{aligned}
& M+k+1 \quad \text { if } k \geqq+0, \\
& L-k+1 \quad \text { if } k \leqq-0 .
\end{aligned}
$$

We see that the elements of $\mathbf{T}_{k}$ have degrees

$$
\begin{aligned}
& k+1, k+1, k+2, k+2, \cdots, k+l+1, k+l+1, \cdots \quad \text { for } k \geqq+0, \\
& -k+1,-k+1,-k+2,-k+2, \cdots,-k+l+1,-k+l+1, \cdots \quad \text { for } k \leqq-0 .
\end{aligned}
$$

The following is trivial.

PROPERTY 7. $d \geqq|k|+1$ iff $\mathbf{T}_{k}$ contains exactly two elements of degree $d$.

That $\mathbf{R} \in \mathbf{T}_{k}$ has degree $d$ means that its elements $\underline{R}=P / Q \in \mathbf{R}$ that are nonnill can be represented by

$$
P=\sum_{j=0}^{d} a_{j} z^{j}, \quad Q=\sum_{j=0}^{j-k-1} b_{j} z^{j} \quad \text { for } k \geqq+0
$$

and

$$
P=\sum_{j=0}^{d-|k|-1} a_{j} z^{j}, \quad Q=\sum_{j=0}^{d} b_{j} z^{j} \quad \text { for } k \leqq-0 .
$$

If the degree is strict for $P$ and/or for $Q$ then we say that the Padé form $R$ has degree $d$.

If $\underline{R} \in \mathbf{R} \in \mathbf{T}_{k}$ has the same degree as $\mathbf{R}$, then we call it a degree representant of $\mathbf{R}$.

Remark that the degree of $\{L / M\}$ is dependent on the staircase of which it is considered to be an element. Since $\{L / M\}$ is always on two neighboring staircases, it has also two possible degrees, but it will be clear from the context which one is meant. 
By writing a subscript for $\mathbf{R}: \mathbf{R}_{d} \in \mathbf{T}_{k}$ we mean that $\mathbf{R}_{d}$ has degree $d$. There are two possible elements that can be indicated by $\mathbf{R}_{d}$ : one that is on the inner diagonal and one that is on the outer diagonal. If we want to make a difference between them then we write $\mathbf{R}_{d}^{\mathrm{I}}$ and $\mathbf{R}_{d}^{\mathrm{O}}$ respectively. put

By Property 7, $\mathbf{R}_{0}$ will never be a member of $\mathbf{T}_{k}$ but for notational comfort we will

$$
\mathbf{R}_{+0}=\left\{\frac{1}{0}\right\} \in \mathbf{T}_{k} \quad \text { for } k \geqq+0 \quad \text { and } \quad \mathbf{R}_{-0}=\left\{\frac{0}{1}\right\} \in \mathbf{T}_{k} \quad \text { for } k \leqq-0 .
$$

Because $c_{0} \neq 0$, its order is always 0 .

Next we will investigate some relations between the order and the degree of some $\mathbf{R} \in \mathbf{T}_{k}$. It follows from the definition that the set $\mathbf{R}$ grows with its degree so we have clearly

Property 8. The order of $\mathbf{R}_{d} \in \mathbf{T}_{k}$ is nondecreasing with its degree.

We will now need the following concepts: $N_{L}=$ the highest possible order obtainable by an element of degree $\leqq L$ (this is the order of $\mathbf{R}_{L}^{\mathrm{O}}$ ) and its inverse is $L_{N}=$ the lowest possible degree allowing an order $\geqq N$ approximation.

These are clearly not 1-1 relations. It is trivially seen that the following is true.

PROPERTY 9.

$$
L_{N+1} \geqq L_{N} \geqq L_{0}=|k| \text {. }
$$

PROPERTY 10. If $\underline{R} \in \mathbf{R}_{L_{N}} \in \mathbf{T}_{k}$ is of order $\geqq N$ then it is a degree representant of $\mathbf{R}_{L_{N}}$ (i.e., it is of degree $L_{N}$ ).

Proof. Clearly if the degree of $\underline{R}$ were $d<L_{N}$ then $L_{N}$ would not be the lowest degree allowing an order $\geqq N$. $\mathbf{R}_{L_{N}}^{\mathrm{O}}$

COROLLARY 1. The order representant of $\mathbf{R}_{L_{N}}^{\mathrm{O}} \in \mathbf{T}_{k}$ is a degree representant of

PROPERTY 11. If $L_{N}<L_{N+1}$ then $\mathbf{R}_{L_{N}} \in \mathbf{T}_{k}$ has an order which is not higher than $N$. The order of $\underline{R}_{L_{N}}^{O}$ is exactly $N$ iff $L_{N}<L_{N+1}$.

Proof. If the order of $\mathbf{R}_{L_{N}}$ were $\geqq N+1$ then we would have that $L_{N+1} \leqq L_{N}$. This proves the first part. Since $\mathbf{R}_{L_{N}}^{\mathrm{I}_{N}} \subset \mathbf{R}_{L_{N}}^{\mathrm{O}}$ we must have by definition of $L_{N}$ that the order of $\mathbf{R}_{L_{N}}^{O}$ is at least $N$. This and the first part give the necessary condition.

By Property 1 we know that $\mathbf{R}_{L_{N}}^{\mathrm{O}}$ contains all rational forms of a degree $\leqq L_{N}$, so the order is the highest obtainable by a degree $\leqq L_{N}$. This means that $L_{N+1}>L_{N}$. This proves the sufficient condition.

CoRollaRY 2. If $L_{N}<L_{N+1}$ and $\underline{R} \in \mathbf{R}_{L_{N}}^{\mathrm{O}} \in \mathbf{T}_{k}$ has an order $N$ then $\underline{R}$ is the order representant of $\mathbf{R}_{L_{N}}^{\mathrm{O}}$.

PROPERTY 12. If $\underline{R}$ exists having degree $L_{N}$ and an order $N^{\prime} \geqq N$ then $L_{N^{\prime}}=L_{N}$.

Proof. The existence of $\underline{R}$ clearly implies that $L_{N^{\prime}} \leqq L_{N}$. It cannot be less because of Property 9.

A proof of the following proposition can be found in [9].

Proposition 1. If there exists a Padé form $\underline{R} \in \mathbf{R} \in \mathbf{T}_{k}$ of degree $p$, having an order $N \geqq p+1$ and if there exists a Padé form $\underline{S}$ having degree $q$ and an order $M \geqq N+1$ then $q \geqq N+|k|+1-p$.

COROLlARY 3. 1. If $L_{N+1}>L_{N}$ then $L_{N+1} \geqq N+|k|+1-L_{N}$.

2. If $\mathbf{R}$ of degree $L_{N}$ and of order $N \geqq L_{N}+1$ exists then

$$
L_{N+1} \geqq \max \left(L_{N}, N+|k|+1-L_{N}\right) .
$$

Proof. 1. By Corollary 1 we know that the order representant of $\mathbf{R}_{L_{N}}^{\mathrm{O}}$ has degree $L_{N}$ and by Property 11 we know that its order is exactly $N$. The order representant of 
$\mathbf{R}_{L_{N+1}}^{O}$ is of an order at least $N+1$ and of degree $L_{N+1}$. Taking these order representants as $R$ and $\underline{S}$ in Proposition 1 we find the desired inequality.

2. Since $\mathbf{R}_{L_{N+1}}^{O}$ has an order representant of degree $L_{N+1}$ (Corollary 1 ) and of an order at least $N+1$. The proposition is again applicable. Together with Property 9 this gives (8).

3. Algorithms. Our aim will be to construct a staircase $\underline{T}_{k}$ with elements of strictly growing order, keeping the degree as low as possible, thus not increasing the degree $L$ until the element of highest possible order $N^{\prime}=N_{L}$ is found. Then we increase the degree from $L$ to $L_{N^{\prime}+1}$ etc.

We directly formulate the algorithm and prove its correctness.

THEOREM 1. For some integer $k \in\{\cdots,-2,-1,-0,+0,1,2, \cdots\}$ construct the staircase $\underline{T}_{k}$ consisting of the elements $\underline{R}^{(i)}, i=1,2, \cdots$ by the following Algorithm A.

Algorithm A.

Initialization:

$$
\begin{aligned}
& d_{0}=0, \quad \underline{R}^{(0)}=\frac{1}{0}(k \geqq+0) \quad \text { or } \quad \frac{0}{1}(k \leqq-0), \quad N_{0}^{\prime}=0 ; \\
& r_{0}=-1(k \geqq+0) \quad \text { or } \quad c_{0}(k \leqq-0) ; \\
& d_{1}=|k|, \quad \underline{R}^{(1)}=[k / 0] \quad(k \geqq+0) \quad \text { or } \quad[0 /-k] \quad(k \leqq-0) ; \\
& N_{1}^{\prime}=\text { the order of } \underline{R}^{(1)} ; \\
& r_{1}=\text { the leading nonzero coefficient of } Z^{(1)} .
\end{aligned}
$$

Recursively for $i=2,3, \cdots$ do the following:

1. Select $\rho_{i}$ by the following criterion.

$\underline{K}: \rho_{i}$ is some $\rho$ such that $N_{\rho}^{\prime}+|k|+1-d_{\rho}=d_{\rho+1}=d_{\rho+2}=\cdots=d_{i-1}$.

2. Form

$$
\begin{aligned}
& P^{(i)}=P^{(i-1)}-F_{i} P^{\left(\rho_{i}\right)} z^{\mu_{i}}, \\
& Q^{(i)}=Q^{(i-1)}-F_{i} Q^{\left(\rho_{i}\right)} z^{\mu_{i}}
\end{aligned}
$$

with $F_{i}=r_{i-1} r_{\rho_{i}}^{-1}$ and $\mu_{i}=N_{i-1}^{\prime}-N_{\rho_{i}}^{\prime}$.

3. For $\underline{R}^{(i)}=P^{(i)} / Q^{(i)}$ compute

$N_{i}^{\prime}=$ the order of $\underline{R}^{(i)}$,

$r_{i}=$ the leading nonzero coefficient of $Z^{(i)}$.

4. Set

$$
N_{i}=N_{i-1}^{\prime}+1 \quad \text { and } \quad d_{i}=\max \left(d_{i-1}, N_{i}+|k|-d_{i-1}\right) .
$$

end of Algorithm A.

Then we have:

1. $N_{i}^{\prime} \geqq N_{i}, i=1,2, \cdots$.

2. $\underline{R}^{(i)}$ is of degree $d_{i}<N_{i}^{\prime}, i=1,2, \cdots$.

3. The outer diagonal of $T_{k}$ is a part of $\underline{T}_{k}$. It is formed by the elements $\underline{R}^{(i)}$ for which $d_{i}<d_{i+1}$. 
Comment. If the theorem is proved then two possible variants of criterion $\underline{K}$ are

$$
\underline{K}_{1}: \rho_{i}=\max _{\rho<i-1}\left\{\rho \mid d_{\rho}<d_{\rho+1}\right\}
$$

or

$$
\underline{K}_{2}: \rho_{i}=\max _{\rho<i-1}\left\{\rho \mid d_{\rho} \leqq d_{\rho+1}\right\}
$$

Proof. The theorem is true for $i=1,2$. For the induction step we have:

1. Because

$$
\begin{aligned}
& Z^{(i-1)}=r_{i-1} z^{N_{i-1}^{\prime}}+\cdots, \\
& Z^{\left(\rho_{i}\right)}=r_{\rho_{i}} z^{N_{\rho_{i}}^{\prime}}+\cdots
\end{aligned}
$$

and

$$
Z^{(i)}=Z^{(i-1)}-r_{i-1} r_{\rho_{i}}^{-1} z^{N_{i-1}^{\prime}-N_{\rho_{i}}^{\prime}} Z^{\left(\rho_{i}\right)},
$$

$\underline{R}^{(i)}$ must be at least of order $N_{i-1}^{\prime}+1=N_{i}$.

2. Let us determine the degree of $\underline{R}^{(i)}$. By construction we have

$$
\text { degree } \underline{R}^{(i)} \leqq \max \left(\text { degree } \underline{R}^{(i-1)}, \mu_{i}+\text { degree } \underline{R}^{\left(\rho_{i}\right)}\right) \text {, }
$$

and by induction hypothesis

$$
\begin{aligned}
& \text { degree } \underline{R}^{(i-1)}=d_{i-1}, \\
& \text { degree } \underline{R}^{\left(\rho_{i}\right)}=d_{\rho_{i}} ;
\end{aligned}
$$

and criterion $\underline{K}$ gives

$$
d_{i-1}=d_{i-2}=\cdots=d_{\rho_{i}+1}=N_{\rho_{i}}^{\prime}+|k|+1-d_{\rho_{i}}
$$

or

$$
d_{\rho_{i}}=N_{\rho_{i}}^{\prime}+|k|+1-d_{\rho_{i+1}},
$$

so that

$$
d_{\rho_{i}}+\mu_{i}=N_{i-1}^{\prime}+|k|+1-d_{i-1} .
$$

So we have that

$$
\text { degree } \underline{R}^{(i)} \leqq \max \left(d_{i-1}, N_{i-1}^{\prime}+|k|+1-d_{i-1}\right)=d_{i} .
$$

Since its order is at least $N_{i}$ we have

$$
L_{N_{i}} \leqq \text { degree } \underline{R}^{(i)} \leqq d_{i}
$$

By induction hypothesis again we have that degree $\underline{R}^{(i-1)}=d_{i-1}=L_{N_{i-1}}$ and by Property 12 this is also $L_{N_{i-1}^{\prime}}$. Its order is $N_{i-1}^{\prime}>L_{N_{i-1}^{\prime}}$ so (because of Corollary 3.2) we have that

$$
\begin{aligned}
L_{N_{i-1}^{\prime}+1} & =L_{N_{i}} \geqq \max \left(L_{N_{i-1}^{\prime}}, N_{i-1}^{\prime}+|k|+1-L_{N_{i-1}^{\prime}}\right) \\
& =\max \left(L_{N_{i-1}}, N_{i-1}^{\prime}+|k|+1-L_{N_{i-1}}\right) .
\end{aligned}
$$

Expressions (9) and (10) then imply that $L_{N_{i}}=$ degree $\underline{R}^{(i)}=d_{i}$.

Point 2 is proved if $d_{i}<N_{i}^{\prime}$. By (10) we know that we have one of the following:

a) $d_{i}=L_{N_{i}}=L_{N_{i-1}^{\prime}}<N_{i-1}^{\prime}$ (by induction);

b) $d_{i}=N_{i-1}^{\prime}+|k|+1-L_{N_{i-1}} \leqq N_{i-1}^{\prime}$ (Property 7).

Thus in both cases we have $d_{i} \leqq N_{i-1}^{\prime}<N_{i}^{\prime}$ while $N_{j}^{\prime}$ is strictly increasing. 
3. Take those $\underline{R}^{(l)}$ such that $d_{l}<d_{l+1}$. By Property 11 we then have that the order or $R_{d_{l}}$ is at most $N_{l}$ but its order is at least $N_{l}$ as proved in the first part of this theorem. We thus have that $N_{l+1}=N_{l}^{\prime}+1=N_{l}+1$. Corollary 2 then says that $\underline{R}^{(l)}$ is the order representant of $\mathbf{R}_{L_{N_{l}}}^{\mathrm{O}}$. Since $N_{l}$ is strictly increasing we have that $\underline{R}^{(l)}$ cannot be duplicated and it must be the element of $T_{k}$ having the order $N_{l}$. Conversely all elements of $T_{k}$ must be part of $T_{k}$ because we cannot jump over one of them by Corollary 3 .

Remark. We could write down directly the recursion between elements $\underline{R}^{(l)}$ that are on the outer diagonal of $T_{k}$.

Set as before $S=\alpha P+\beta Q$ and let

$$
d_{m}<d_{m+1}=d_{m+2}=\cdots=d_{i}<d_{i+1}=d_{i+2}=\cdots=d_{j}<d_{j+1} .
$$

For simplicity let us take criterion $\underline{K}_{2}$; then

$$
\rho_{l}=m \quad \text { for } l=m+2, \cdots, i+1,
$$

and

$$
\begin{aligned}
& \quad \rho_{l}=i \quad \text { for } l=i+2, \cdots, j+1 \\
S^{(j)}= & S^{(j-1)}-F_{j} z^{\mu_{i}} S^{(i)} \\
= & S^{(j-2)}-\left(F_{j-1} z^{\mu_{i-1}}+F_{j} z^{\mu_{j}}\right) S^{(i)} \\
= & \cdots \\
= & S^{(i+1)}-\left(F_{i+2} z^{\mu_{i+2}}+\cdots+F_{j} z^{\mu_{j}}\right) S^{(i)} \\
= & S^{(i)}-F_{i+1} z^{\mu_{i+1}} S^{(m)}-\left(F_{i+2} z^{\mu_{i+2}}+\cdots+F_{j} z^{\mu_{i}}\right) S^{(i)} \\
= & \left(1-F_{i+2} z^{\mu_{i+2}}-\cdots-F_{j} z^{\mu_{j}}\right) S^{(i)}-F_{i+1} z^{\mu_{i+1}} S^{(m)}
\end{aligned}
$$

Equation (11) can be thought of as the forward recursion for the evaluation of a continued fraction whose convergents are the successive outer diagonal elements of $T_{k}$, e.g., for $k \geqq+0$,

$$
[k / 0]+\sum_{l} \frac{\phi_{l}(z) \mid}{\mid \psi_{l}(z)}-\frac{F_{i+1} z^{\mu_{i+1}}}{\sqrt{1-F_{i+2} z^{\mu_{i+2}}-\cdots-F_{j} z^{\mu_{j}}}+\cdots}+\cdots
$$

This is a continued fraction like those mentioned in [11], [2].

Proposition 2. Applying Algorithm A in the normal case we have for $i \geqq 2$ :

1) $d_{i-1} \leqq d_{i} \leqq d_{i-1}+1$ (i.e., $\left.N_{i-1}^{\prime}+|k| \leqq 2 d_{i-1}\right)$.

2) If $d_{i}=d_{i-1}$ then $N_{i}^{\prime}=N_{i}=2 d_{i}-|k|$,

$$
\begin{aligned}
& \mu_{i}=1, \\
& d_{i+1}=d_{i}+1 .
\end{aligned}
$$

If $d_{i}=d_{i-1}+1$ then $2 d_{i}-|k|-1 \leqq N_{i}^{\prime} \leqq 2 d_{i}-|k|$.

The verification is left to the reader.

Suppose that in the normal case we have that

$$
d_{i-2}<d_{i-1}=d_{i}<d_{i+1}=d_{i+2}
$$

then if Algorithm A is used with criterion $\underline{K}_{1}$

$$
\begin{aligned}
S^{(i+2)} & =S^{(i+1)}-r_{i+1} r_{i}^{-1} z S^{(i)} \\
& =S^{(i)}-r_{i} r_{i-2}^{-1} z^{2} S^{(i-2)}-r_{i+1} r_{i}^{-1} z S^{(i)} \\
& =\left(1-r_{i+1} r_{i}^{-1} z\right) S^{(i)}-r_{i} r_{i-2}^{-1} z^{2} S^{(i-2)}
\end{aligned}
$$


Expressing $r_{i+1}$ as a function of residual coefficients of $Z^{(i-2)}$ and $Z^{(i)}$ we find that this is the method of Brezinski. Expression (11) can be seen as a generalization of (12) to the nonnormal case. If Algorithm A is used with criterion $\underline{K}_{2}$ then we find the WatsonThacher recursions.

We will now specify $K_{3}$ as a special form of criterion $\underline{K}$ and prove that not only the outer diagonal of $T_{k}$ is constructed, but also the inner one.

$\underline{K}_{3}$ : For $i=2,3, \cdots$ use

$$
\underline{K}_{1}: \rho_{i}=\max _{\rho<i-1}\left\{\rho \mid d_{\rho}<d_{\rho+1}\right\}
$$

to select $\rho_{i}$ until $d_{i}<d_{i+1}$. From then on use

$$
K_{2}^{\prime}: \rho_{i}=\max _{\rho<i-1}\left\{\rho \mid d_{\rho} \leqq d_{\rho+1} \text { and } \underline{R}^{(\rho)} \in T_{k}\right\}
$$

until $N_{j-1}^{\prime} \geqq 2 d_{j-1}-|k|-1$. From then on use again $\underline{K}_{1}$ etc.

THEOREM 2. If in Algorithm A we take $\underline{K}_{3}$ as a special case of $K$ and call the corresponding algorithm A3 then:

1. The statements of Theorem 1 are valid for Algroithm A3.

2. The last elements $\underline{R}^{(i)}$ for which $\rho_{i}$ is selected by $\underline{K}_{1}$ before a switch to $\underline{K}_{2}^{\prime}$ form the outer diagonal of $T_{k}$.

3. The last elements $\underline{R}^{(i)}$ for which $\rho_{i}$ is selected by $\underline{K}_{2}^{\prime}$ before a switch to $\underline{K}_{1}$ form the inner diagonal of $T_{k}$.

Proof. (for $k \geqq+0$ ). Clearly we only have to prove condition 3 . Therefore we will first prove the following lemma.

LEMMA. If $\rho_{i}$ is selected by $\underline{K}_{2}^{\prime}$ then $P^{(i)}$ (the numerator of $\underline{R}^{(i)}$ ) is of degree $d^{\circ} P^{(i)} \leqq d_{i}-1$ (i.e., $\underline{R}^{(i)} \in \mathbf{R}_{d_{i}}^{\mathrm{I}}$ ).

Proof (for $k \geqq+0) . \underline{R}^{(1)}=[k / 0]$ is of degree $k+1$ and $d^{\circ} P^{(1)} \leqq k$. Suppose that

$$
d_{2}=d_{3}=\cdots=d_{i}<d_{i+1}
$$

then $\rho_{2}, \cdots, \rho_{i}$ are selected by $\underline{K}_{1}$ and $\rho_{i+1}$ by $\underline{K}_{2}^{\prime} .1 / 0$ is the first member of $T_{k}$ (outer diagonal) and $[k / 0]$ is the second (inner diagonal) and by Theorem $1, \underline{R}^{(i)}$ must be the third (outer diagonal). $\underline{K}_{2}^{\prime}$ then gives $\rho_{i+1}=1$. By construction we then have that $d^{\circ} P^{(i+1)} \leqq d_{i+1}-1$. If $N_{i+1}^{\prime}<2 d_{i+1}-k-1$ then $\rho_{i+2}$ is again selected by $K_{2}^{\prime}$ and thus again $d^{\circ} P^{(i+2)} \leqq d_{i+2}-1$. These are the first steps of an induction proof along the idea that if $\rho_{i}$ is selected by $K_{2}^{\prime}$ then $P^{(i)}$ is formed from $P^{(i-1)}$ and $P^{\left(\rho_{i}\right)}$, and at least one of $\rho_{i-1}$ or $\rho_{\rho_{i}}$ was selected by $K_{2}^{\prime}$. The induction hypothesis then proves the lemma.

$\underline{K}_{2}^{\prime}$ is used as long as $N_{j-1}^{\prime}<2 d_{j-1}-k-1$ or equivalently $d_{j}=d_{j-1}$. Thus during a " $K_{2}^{\prime}$ session" we see that the degree remains constant: say $d$. By the lemma we know that all the corresponding $\underline{R}^{(j)}$ are in $\{d-1 / d-k-1\}$. From the moment that $N_{1}^{\prime} \geqq$ $2 d-k-1$ we see that it must be the Padé approximant of $\{d-1 / d-k-1\}$. So it is an inner diagonal element of $T_{k}$. From then on we look for an outer diagonal element of $T_{k}$, using $K_{1}$. Owing to Corollary 3.2 we will find all the elements.

Proposition 3. If the Padé table is normal then for Algorithm A3 we have in addition to the statements of Theorem 2 that

$$
d_{i}=d_{i-1}+1 \quad \text { and then } \quad N_{i}^{\prime}=N_{i}=2 d_{i}-|k|-1, \quad d_{i+1}=d_{i}
$$

or

$$
d_{i}=d_{i-1} \quad \text { and then } \quad N_{i}^{\prime}=N_{i}=2 d_{i}-|k|, \quad d_{i+1}=d_{i}+1
$$

and always $\rho_{i}=i-2$ and $\mu_{i}=1$ (except $\mu_{2}$ which is $\left.|k|+1\right)$.

$\underline{K}_{1}$ and $\underline{K}_{2}^{\prime}$ are used alternatively.

Proof. The proof is trivial and is left to the reader. 
Proposition 3 implies that for all $i$ we have

$$
S^{(i)}=S^{(i-1)}-r_{i-1} r_{i-2}^{-1} z S^{(i-2)} \text {. }
$$

This is the recursion of Watson's and Thacher's algorithm.

Additional remarks. 1) If we drop the subscript $i$ everywhere and if we have that

$$
\underline{R}=\frac{P}{Q} \quad \text { and } \quad Q=\sum_{l=0}^{d} b_{l} z^{l} \quad\left(b_{0}=1\right),
$$

then we can compute $N^{\prime}$ and $r$ by the following algorithm.

Algorithm.

1. $K=N$.

2. Compute

$$
S_{K}=\sum_{l=0}^{d} b_{l} c_{K-l}
$$

3. If $s_{K}=0$ then $K=K+1$ and go to 2 .

$$
\text { else } r=s_{K} \text { and } N^{\prime}=K \text {. }
$$

If there is no finite $K$ for which $s_{K} \neq 0$ then $f(z)=\underline{R}$.

2) If we only want to compute $[L / M]$ for certain prespecified values of $L$ and $M$ then we need not compute all the numerators of the elements preceding $[L / M]$ in the staircase $\underline{T}_{k}$. It is sufficient to use only the recursion for $Q^{(i)}$ and find

$$
P^{[L / M]}=\sum_{l=0}^{L} a_{l}^{[L / M]} z^{l}
$$

from

$$
a_{l}^{[L / M]}=\sum_{j=0}^{M} b_{j}^{[L / M]} c_{l-j}, \quad l=0,1, \cdots, L .
$$

3) Another possibility inspired by the Thacher algorithm would be to compute only $F_{i}$ using the recursions for $S^{(i)}=Z^{(i)}$,

$$
Z^{(i)}=Z^{(i-1)}-F_{i} z^{\mu_{i}} Z^{\left(\rho_{i}\right)}
$$

This would correspond to a continued fraction-like approach and is most comfortably found as a generalization of the Viskovatoff division algorithm as is done in [11], [2]. In [3] it is explained that such an approach corresponds to the triangular factorization of a Hankel matrix while the direct treatment of this paper actually gives a fast algorithm for the factorization of the inverse of the same Hankel matrix.

4. Examples. Take

$$
f(z)=\frac{1-z+z^{3}}{(1-z)^{2}}=1+z+z^{2}+2 z^{3}+3 z^{4}+\cdots+n z^{n+1}+\cdots .
$$

Algorithm A with $\underline{K}=\underline{K}_{1}$ would give us Table 1 for $T_{-0}$ (the outer diagonal elements of $T_{k}$ are marked with $O$ ). 
TABLE 1

\begin{tabular}{ccccccc}
\hline$i$ & 0 & 1 & 2 & 3 & 4 & 5 \\
\hline$\underline{R}^{(i)}$ & $\frac{0}{1}$ & $\frac{1}{1}$ & $\frac{1}{1-z}$ & $\frac{1}{1-z-z^{3}}$ & $\frac{1+z^{3}}{1-z-z^{4}}$ & $\frac{1-z+z^{3}}{1-2 z+z^{2}}$ \\
\hline$N_{i}^{\prime}$ & 0 & 1 & 3 & 6 & 7 & $\infty$ \\
\hline$d_{i}$ & 0 & 1 & 1 & 3 & 4 & 4 \\
\hline$r_{i}$ & 1 & 1 & 1 & -1 & -1 & 0 \\
\hline $\operatorname{diag}$ & $O$ & & $O$ & $O$ & & $O$ \\
\hline
\end{tabular}

Criterion $\underline{K}_{2}$ would give us Table 2 .

TABLE 2

\begin{tabular}{ccccccc}
\hline$i$ & 0 & 1 & 2 & 3 & 4 & 5 \\
\hline $\boldsymbol{R}^{(i)}$ & $\frac{0}{1}$ & $\frac{1}{1}$ & $\frac{1}{1-z}$ & $\frac{1-z^{2}}{1-z-z^{2}}$ & $\frac{1}{1-z-z^{3}}$ & $\frac{1-z+z^{3}}{1-2 z+z^{2}}$ \\
\hline $\boldsymbol{N}_{i}^{\prime}$ & 0 & 1 & 3 & 5 & 6 & $\infty$ \\
\hline$d_{i}$ & 0 & 1 & 1 & 3 & 3 & 4 \\
\hline$r_{i}$ & 1 & 1 & 1 & -1 & -1 & 0 \\
\hline $\operatorname{diag}$ & $O$ & & $O$ & & $O$ & $O$ \\
\hline
\end{tabular}

Criterion $\underline{K}_{3}$ would then give the same as $\underline{K}_{2}$ (for this example).

TABLE 3

\begin{tabular}{ccccccc}
\hline$\underline{K}_{i}$ & & & $\underline{\underline{K}}_{1}$ & $\underline{\underline{K}}_{2}^{\prime}$ & $\underline{\boldsymbol{K}}_{1}$ & $\underline{\boldsymbol{K}}_{2}^{\prime}$ \\
\hline $\operatorname{diag}$ & $O$ & $I$ & $O$ & $I$ & $O$ & $I$ \\
\hline
\end{tabular}

That we cannot replace $K_{2}^{\prime}$ by $\underline{K}_{2}$ in Algorithm A3 may be shown by the following example

$$
f(z)=\frac{1+z^{4}}{1-z^{5}}=1+z^{4}+z^{5}+z^{9}+z^{10}+2 z^{15}+2 z^{20}+\cdots
$$

$T_{+0}$ computation by Algorithm A3 gives us Tables 4 and 5 . 
TABLE 4

\begin{tabular}{ccccccc}
\hline$i$ & 0 & 1 & 2 & 3 & 4 & 5 \\
\hline$\underline{R}^{(i)}$ & $\frac{1}{0}$ & $\frac{1}{1}$ & $\frac{1+z^{4}}{1}$ & $\frac{1-z+z^{4}}{1-z}$ & $\frac{1-z+z^{2}+z^{4}}{1-z+z^{2}}$ & $\frac{1-z+z^{2}-z^{3}+z^{4}}{1-z+z^{2}-z^{3}}$ \\
\hline$N_{i}^{\prime}$ & 0 & 4 & 5 & 6 & 7 & 8 \\
\hline$d_{i}$ & 0 & 1 & 4 & 4 & 4 & 4 \\
\hline$r_{i}$ & -1 & 1 & 1 & -1 & 1 & -1 \\
\hline$\underline{K}_{i}$ & & & $\underline{K}_{1}$ & $\underline{K}_{1}$ & $K_{1}$ & $O$ \\
\hline $\operatorname{diag}$ & $O$ & $I$ & & & & $K_{1}$ \\
\hline
\end{tabular}

TABLE 5

\begin{tabular}{cccc}
\hline$i$ & 6 & 7 & 8 \\
\hline$\underline{R}^{(i)}$ & $\frac{1-z+z^{2}-z^{3}+2 z^{4}}{1+z+z^{2}-z^{3}+z^{4}}$ & $\frac{1+z-z^{2}+z^{3}+2 z^{5}}{1+z-z^{2}+z^{3}-z^{4}}$ & $\frac{1+z^{4}}{1-z^{5}}$ \\
\hline$N_{i}^{\prime}$ & 9 & 10 & $\infty$ \\
\hline$d_{i}$ & 5 & 5 & 6 \\
\hline$r_{i}$ & 2 & 2 & 0 \\
\hline$\underline{K}_{i}$ & $\underline{K}_{2}^{\prime}$ & $\underline{K}_{1}^{\prime}$ & $\boldsymbol{K}_{2}^{\prime}$ \\
\hline $\operatorname{diag}$ & $I$ & $O$ & $I$
\end{tabular}

If $\underline{K}_{2}$ had been used instead of $\underline{K}_{2}^{\prime}$ then $\underline{R}^{(6)}$ would have been

$$
\underline{R}^{(6)}=\frac{1+z^{4}+z^{5}}{1} \text { and } \quad \underline{R}^{(7)}=\frac{1+z-z^{2}+z^{4}+2 z^{5}}{1+z-z^{2}+z^{3}-z^{4}}
$$

$\underline{R}^{(7)}$ is on the outer diagonal so the inner diagonal element is jumped over.

Acknowledgment. We thank Professor P. Dewilde who introduced the Berlekamp-Massey algorithm to us and Professor L. Wuytack who brought the papers of Bussonnais and Cordellier to our attention.

\section{REFERENCES}

[1] C. Brezinski, Computation of Padé approximants and continued fractions, J. Comput. Appl. Math., 2 (1976), pp. 113-123.

[2] A. Bultheel, Division algorithms for continued fractions and the Padé table, Applied Math. and Programming Division, Rep. TW 41, Katholieke Universiteit Leuven, Belgium, August 1978.

[3] A. Bultheel, Fast algorithms for the factorization, of Hankel and Toeplitz matrices and the Padé approximation problem, Applied Math. and Programming Division, Rep. TW 42, Katholieke Universiteit Leuven, Belgium, October 1978. 


\section{A. BULTHEEL}

[4] D. Bussonnais, "Tous" les algorithmes de calcul par recurrence des approximants de Padé d'une serie, Construction de fractions continues correspondantes, Séminaire d'Analyse Numérique, no. 293, Grenoble, France, 1978.

[5] G. Claessens, A new look at the Padé table and the different methods for computing its elements, J. Comput. Appl. Math., 1 (1975), pp. 141-152.

[6] B. Cordellier, Deux algorithmes de calcul récursif des éléments d'une table de Padé non normale, Conference on Padé approximation, Lille, France, 1978.

[7] L. S. DE JONG, Numerical aspects of realization algorithms in linear systems theory, Ph.D. thesis, T. H. Eindhoven, The Netherlands, 1975.

[8] W. B. GRAGG, The Padé table and its relation to certain algorithms of numerical analysis, SIAM Rev., 14 (1972), pp. 1-62.

[9] J. L. MASSEY, Shift-register synthesis and BCH decoding, IEEE Trans. Information Theory, IT-15 (1969), pp. 122-127.

[10] R. J. MCEliece AND J. B. Shearer, A property of Euclid's algorithm and an application to Padé approximation, this Journal, 34 (1978), pp. 611-616.

[11] J. A. MURPHY AND H. R. O'DonOHOE, A class of algorithms for obtaining rational approximants to functions which are defined by power series, Z. Angew. Math. Phys., 28 (1977), pp. 1121-1131.

[12] P. J. S. WATSON, Algorithms for differentiation and integration, Padé Approximants and Their Applications, P. R. Graves-Morris, ed., Academic Press, London, 1973, pp. 93-98. 